NOTA TÉCNICA:

\title{
DESENVOLVIMENTO DE DISPOSITIVO ELETRÔNICO PARA MARCAÇÃO AUTOMÁTICA DO PONTO DE TRAÇAMENTO DA MADEIRA POR UMA GARRA TRAÇADORA
}

\author{
Haroldo Carlos Fernandes ${ }^{1}$, Sebastião Eudes Lopes ${ }^{2}$, Mauri Martins Teixeira ${ }^{3}$, Luciano José Minette ${ }^{4}$, \\ Paula Cristina Natalino Rinaldi ${ }^{5}$
}

\begin{abstract}
RESUMO
Este trabalho foi conduzido em povoamentos de eucalipto de uma empresa florestal do Estado de Goiás, com o objetivo de desenvolver dispositivo eletrônico para marcação automática do ponto de traçamento da madeira. O trator utilizado neste trabalho foi uma escavadora hidráulica, modelo $320 \mathrm{CL}$, fabricante caterpillar, com $103 \mathrm{~kW}$ de potência nominal do motor. Para o controle do ponto de traçamento dos toretes, foram utilizados um contador controlador programável, um gerador de pulsos e um sensor de proximidade do tipo indutivo. De acordo com os resultados, conclui-se que os valores encontrados para os comprimentos dos toretes quando medidos pelo dispositivo eletrônico e pela tora padrão ficaram próximos do desejado. O sensor utilizado não foi capaz de zerar automaticamente os valores quando o operador ultrapassou a medida desejada. O dispositivo ainda necessita de ajustes principalmente quanto ao tempo de traçamento.
\end{abstract}

Palavras-chave: colheita florestal, processamento, sensores.

\section{ABSTRACT \\ DEVELOPMENT OF AN ELECTRONIC DEVICE FOR AUTOMATIC DEMARCATION OF WOOD BUCKING POINT FOR A BUCKING CLUTCHES}

The study was carried out in eucalyptus plantations of a forest company in the State of Goiás to develop an electronic device to automatically demarcate wood bucking point. The tractor used was a Caterpillar $320 \mathrm{CL}$ with hydraulic excavator and $103 \mathrm{~kW}$ engine. A programmable counter, an impulse generator and an inductive type proximity sensor held in a metal housing were used to control the bucking length. The results showed that the predetermined log lengths measured by the electronic device and standard log were close. The sensor was incapable of resetting automatically if the operator surpassed the required length. The device however requires adjustment, especially for demarcation time.

Keywords: forest harvest, processing, sensors.

\section{Recebido para publicação em 16/03/2010. Aprovado em 14/09/2011.}

1- Eng ${ }^{\circ}$ Agrícola, Professor Associado do Departamento de Engenharia Agrícola da Universidade Federal de Viçosa-MG, email: haroldo@ufv.br

2- Eng ${ }^{\circ}$ Mecânico, DS em Engenharia Agrícola - Mecanização Agrícola, UFV, Viçosa - MG, email: industria@jatiboca.com.br

3- Eng ${ }^{\circ}$ Agrônomo, Professor Associado do Departamento de Engenharia Agrícola da Universidade Federal de Viçosa - MG, email: mauri@ufv.br

4- Professor Adjunto. Departamento de Engenharia Elétrica e de Produção, UFV, Viçosa, MG

5- Eng ${ }^{a}$.Agrícola, Doutoranda em Engenharia Agrícola - Mecanização Agrícola, UFV, Viçosa - MG, email: pcnrinaldi@yahoo.com.br

536 REVENG

536-540 p. Engenharia na AGRicultura, VIÇOSA - MG, V.19 N.6, NOVEMBRO / DEZEMBRO 2011 


\section{INTRODUÇÃO}

A pesquisa a respeito dos fatores humanos e das condições de trabalho nas empresas florestais tem por objetivo aperfeiçoar métodos e técnicas operacionais, de modo a assegurar condições confortáveis e saudáveis no ambiente de trabalho. O conhecimento dessas condições de vida e a busca constante de sua melhoria influenciam diretamente a satisfação do trabalhador, levando ao aumento da produtividade e da qualidade do trabalho (GRANDJEAN, 1998; IIDA, 1990; FIEDLER, 1995; MINETTE, 1996; ANDRADE, 1998).

A mão de obra é um componente essencial para o trabalho florestal, notadamente nas atividades de elevada exigência física, como as operações de colheita, realizadas de forma manual ou semimecanizada, em que se faz uso intensivo de mão de obra. Para caracterizar a mão de obra e as condições de trabalho, é preciso conhecer os fatores humanos relacionados aos trabalhadores, bem como as condições de trabalho, saúde, alimentação, treinamento e segurança (MINETTE et al., 1999). O conhecimento desses fatores é fundamental para que a área de trabalho, o seu arranjo, os equipamentos e as ferramentas sejam bem adaptados às capacidades psicofisiológicas, antropométricas e biomecânicas do trabalhador (MACHADO et al., 1991).

A colheita mecanizada foi a fase do processo produtivo florestal que mais sofreu alterações pela introdução dos tratores florestais para o corte e a extração da madeira. Nas atividades da colheita, os operadores ficam expostos às condições adversas vindas do meio ambiente, como temperatura, poeira, umidade etc., e da máquina, como ruídos, vibrações, postura, gases, temperatura etc., sendo as últimas condições decorrentes do projeto da máquina.

No início do desenvolvimento das máquinas florestais, os projetos estavam mais voltados para a utilização e redução dos custos de produção do equipamento. Entretanto, com o desenvolvimento tecnológico, passou-se a buscar o aperfeiçoamento da relação homem-máquina com a finalidade de impor ao homem uma carga de trabalho mais suave, visando à redução da fadiga e, em consequência, ao aumento da produtividade do conjunto e à melhoria do serviço executado (MENEZEZ et al., 1985).

Atualmente existem várias empresas trabalhando em projetos de alta tecnologia, pensando sempre no operador, pois vale mencionar que o operador, em alguns casos, pode limitar a eficiência de certas operações em até $90 \%$, havendo, por isso, muitos esforços no sentido de aumentar seu conforto. Hoje é comum, em tratores e colhedoras florestais, o controle automático da temperatura e umidade do ar, além dos computadores de bordo para permitir um controle do ponto de traçamento da madeira, controle da produção, comandos ergonômicos e modernos sistemas de suspensão. O uso de máquinas mais confortáveis proporciona um melhor ambiente de trabalho e pode-se atingir um melhor índice de produtividade.

$\mathrm{O}$ uso de sensores permite que uma máquina possa interagir com o ambiente de trabalho de forma flexível. Isto não acontece nas operações pré-programadas em que a máquina realiza tarefas repetitivas por meio de um conjunto de funções programadas. O uso da tecnologia dos sensores introduz nas máquinas uma maior eficiência para lidar com o seu meio e é objeto de uma pesquisa intensa no campo da robótica. Os sensores de estado externos atuam na detecção de variáveis como distância, proximidade e tato, podendo ser classificados em sensores de contato ou não. Os sensores de contato reagem com o contato físico como o toque, com o momento das forças, com o deslizar de materiais. Já os sensores que não são de contato se baseiam nas variações acústicas e nas radiações eletromagnéticas.

As colhedoras florestais, apesar de diferirem em sua construção, de acordo com os fabricantes e sua utilização final, são constituídas basicamente de um conjunto de corte e/ou processamento, corte, processamento e movimentação. $\mathrm{O}$ conjunto de corte e processamento é responsável pela operação de "derrubada" da árvore, desgalhamento e traçamento, dependendo do cabeçote. O corte, propriamente dito, pode ser realizado com lâminas de corte ou correntes semelhantes 
às usadas em motosserras. O conjunto de corte e processamento é sustentado por um braço, acionado hidraulicamente, que coloca o cabeçote de corte e ou processamento junto à árvore a ser derrubada, para a realização do corte e das operações subsequentes. $\mathrm{O}$ conjunto de movimentação é encarregado de movimentar e sustentar os conjuntos de corte e de processamento, podendo ser montado sobre esteiras ou rodas. Assim, o sistema de colheita pode ter equipamentos que cortam, desgalham, separam em toras e empilham na beira da estrada ou ainda aqueles que transportam a madeira (MENDONÇA FILHO, 2000).

Baseando-se neste contexto e considerando que o processamento da madeira é realizado por escavadora hidráulica, adaptada com uma garra traçadora, necessita-se de uma padronização do tamanho das toras a serem traçadas, sendo assim, a empresa faz uso de um gabarito feito com tora padrão, cortada com dimensões entre $5,85 \mathrm{~m}$ a $6,15 \mathrm{~m}$, que serve de referência para o operador traçar os toretes. Este trabalho foi conduzido com objetivo de desenvolver um dispositivo capaz de apontar com precisão ao operador o ponto para traçamento da madeira pela garra traçadora.

\section{MATERIAL E MÉTODOS}

O trabalho foi conduzido em povoamentos florestais de uma empresa Mineradora do grupo Anglo-América na região de Niquelândia, estado de Goiás. O município está situado a $14^{\circ}$ $28^{\prime} 26^{\prime \prime}$ 'de latitude sul e $48^{\circ} 27^{\prime} 35^{\prime}$ " de longitude oeste, com $583 \mathrm{~m}$ de altitude. Os povoamentos florestais são da espécie Eucalyptus grandis, com idade média de 10 anos, espaçamento de $3 \mathrm{x}$ $3 \mathrm{~m}$ e produtividade média de $350 \mathrm{~m}^{3} / \mathrm{ha}$.

O tipo de solo predominante na região é o Latossolo Vermelho-Escuro (LE), álico, Horizonte A moderado, com textura de média a arenosa e relevo plano a suave ondulado. O clima é tropical úmido, com temperatura média em torno de $32^{\circ} \mathrm{C}$.

$\mathrm{O}$ trator utilizado neste trabalho foi uma escavadora hidráulica, modelo $320 \mathrm{CL}$, fabricante caterpillar, com $103 \mathrm{~kW}$ de potência nominal do motor. Na Figura 1é mostrada a escavadora hidráulica adaptada com a garra traçadora.

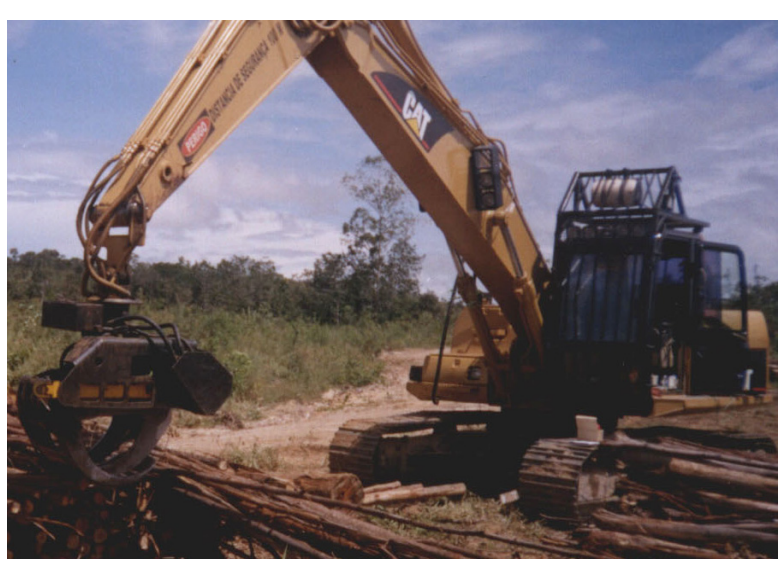

Figura 1. Vista geral da garra traçadora.

O ciclo de trabalho da garra traçadora compreendia buscar, posicionar para o corte, cortar e empilhar o feixe de madeira para o posterior carregamento. Para o corte da madeira, a garra se deslocava em cima de um gabarito com dimensão conhecida para o operador ter noção do comprimento a ser traçado.

O trabalho compreendeu duas etapas básicas:

Primeira etapa: Determinar o volume de madeira a ser traçado no sistema atual e o tempo gasto, incluindo o tempo para posicionamento da tora padrão.

Segunda etapa: Determinar o raio de giro da máquina que permitisse o traçamento, conforme as dimensões estabelecidas. Foi avaliada a distância entre o braço e a lança da máquina, de modo que o comprimento de giro fosse medido pelo dispositivo eletrônico. Para essa leitura, foram relacionados a distância entre os parafusos que sustentam a base da máquina e o local de instalação do sensor com o giro da máquina. Esses parafusos que sustentam a base da máquina estão distanciados $0,12 \mathrm{~m}$ entre si. O sensor, ao detectar o parafuso, envia um sinal ao contador programável que faz a leitura, indicando se o comprimento desejado foi alcançado para que seja efetuado o traçamento dos toretes.

Para o controle do ponto de traçamento dos toretes, foram utilizados um contador controlador programável, linha TP 02, marca WEG, que funciona através de um gerador de pulsos baseado em sensor de proximidade do tipo indutivo, modelo M 12 X 1 CC, com invólucro metálico, proteção contra curto-circuito e rearme automático. O sensor, ao detectar os parafusos mediante o giro da máquina, gera pulsos que são enviados a um contador eletrônico programável de marca turck, modelo NC 400, que possui indicação por display

\section{REVENG


de leds com 5 dígitos numéricos.

A posição do sensor, que era inicialmente vertical à base da máquina, foi alterado para a posição horizontal devido à incidência de erros causados pela distância dos parafusos ao sensor (Figura 2).

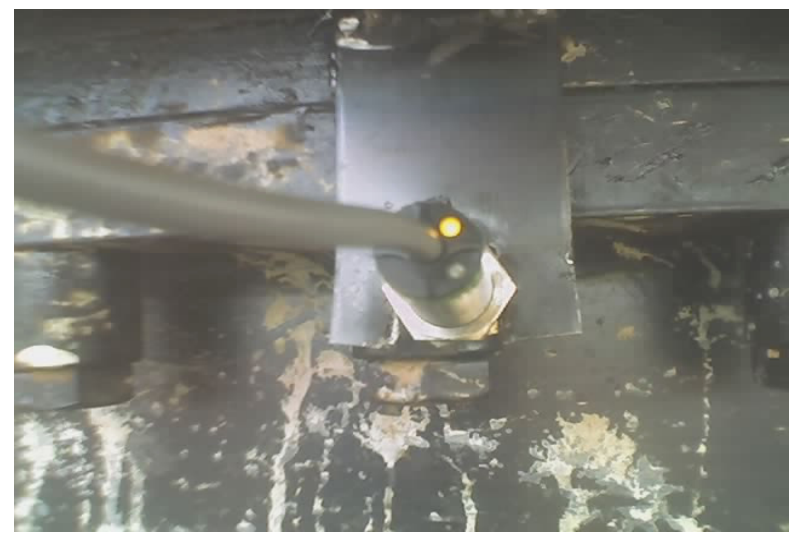

Figura 2. Posição do sensor instalado na base da máquina.

O diagrama do funcionamento do sistema de traçamento dos toretes é apresentado na Figura 3.

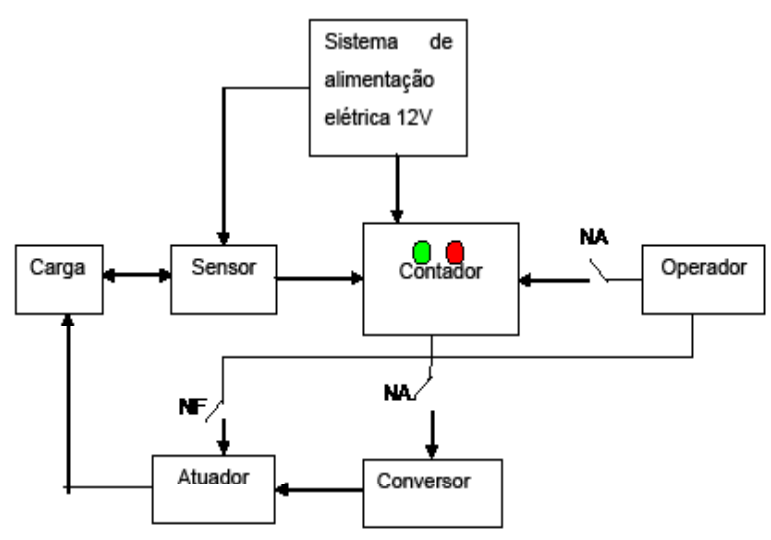

Figura 3. Diagrama de funcionamento do sistema.
O contador programável foi instalado no interior da cabine da máquina de modo a facilitar a visualização da leitura e do ponto de traçamento da madeira. O dispositivo eletrônico foi calibrado utilizando a tora padrão e, ao atingir 6 metros, o visor indica essa leitura, monitorando assim o tempo gasto para o traçamento.

\section{RESULTADOS E DISCUSSÃO}

O dispositivo eletrônico foi calibrado utilizando a tora padrão como referência. Foi necessário fixar a posição do braço e da lança, de modo a formar um ângulo de $42,3^{\circ}$ entre a base da máquina e o ponto de traçamento.

Os comprimentos dos toretes traçados e medidos pela garra com os procedimentos da tora padrão e com o dispositivo eletrônico são mostrados no Quadro 1.

A média dos valores encontrados foi de 5,91 $\mathrm{m}$ quando medidos pelo dispositivo e de $6,03 \mathrm{~m}$ quando medidos com a tora padrão. O coeficiente de variação $(\mathrm{CV})$ para os valores medidos pelo dispositivo eletrônico e para a tora padrão foi de de 6,30 e $6,28 \%$, respectivamente. Os valores encontrados para o comprimento dos toretes não apresentaram diferença estatística, ficando próximos do valor desejado, $6 \mathrm{~m}$.

Os tempos médios gastos para o traçamento medidos pelo dispositivo eletrônico e pela tora padrão foram de 105,11 e 19,22 s, respectivamente. Os valores encontrados para o tempo de traçamento apresentaram diferença estatística a $5 \%$ de probabilidade pelo teste Tukey. O tempo gasto foi devido ao retorno da garra, quando o sensor não

Quadro 1. Tempos, comprimento médios e máximos, desvio padrão e coeficiente de variação dos toretes traçados pela garra traçadora

\begin{tabular}{ccccc}
\hline Parâmetros & $\begin{array}{c}\text { Comprimento dos toretes } \\
\text { traçados e medidos c/ } \\
\text { dispositivo eletrônico (m) }\end{array}$ & Tempo (s) & $\begin{array}{c}\text { Comprimento dos toretes } \\
\text { traçados e medidos c/ tora } \\
\text { padrão (m) }\end{array}$ & Tempo (s) \\
\hline Máximo & 6,50 & 236,00 & 6,70 & 52,00 \\
Mínimo & 5,20 & 36,00 & 4,90 & 8,00 \\
Médio & 5,91 & 105,11 & 6,03 & 19,22 \\
\hline Desvio Padrão & 0,37 & 39,66 & 0,38 & 9,81 \\
\hline CV $(\%)$ & 6,30 & 37,73 & 6,28 & 51,06 \\
\hline
\end{tabular}


atuava ou quando ele ultrapassava o ponto desejado para o traçamento, obrigando assim o operador a zerar o dispositivo eletrônico e iniciar todo o processo.

O ângulo fixado para traçamento ajudou a contribuir para o aumento do tempo, pois a dimensão variava quando o operador instintivamente alterava o ângulo, mexendo no braço ou lança da máquina.

\section{AGRADECIMENTO}

Agradecemos a Fapemig, Fundação de Amparo a Pesquisa do Estado de Minas Gerais, pelo apoio para a realização deste trabalho.

\section{CONCLUSÕES}

- Os valores encontrados para os comprimentos dos toretes, quando medidos pelo dispositivo eletrônico e pela tora padrão, ficaram próximos do desejado;

- Os valores de tempo não foram satisfatórios, sendo os valores medidos pelo dispositivo superiores aos medidos com a tora padrão; e

- O dispositivo, apesar do bom funcionamento, ainda necessita de ajustes, principalmente quanto ao tempo de traçamento.

\section{REFERÊNCIAS BIBLIOGRÁFICAS}

ANDRADE, S.C. Avaliação técnica, social, econômica e ambiental de dois subsistemas de colheita florestal no Litoral Norte da Bahia. Viçosa, MG: UFV, 1998. 125p. Dissertação (Mestrado em Ciência Florestal) - Universidade Federal de Viçosa, 1998.

FIEDLER, N.C. Avaliação ergonômica de máquinas utilizadas na colheita de madeira. Viçosa, MG: UFV, 1995. 126p. Dissertação (Mestrado em Ciência Florestal) Universidade Federal de Viçosa, 1995.

GRANDJEAN, E. Manual de Ergonomia, Adaptando o trabalho ao homem, Editora Artes Médicas Sul Ltda, 1998. 223p.

IIDA, I. Ergonomia: projeto e produção. São Paulo: Edgard Blücher, 1990. 465p.

MACHADO, C.C.; SOUZA, A.P.; LEITE, M. Análise do desempenho de diferentes veículos de transporte florestal rodoviário. Revista Árvore, v.15, n.1, p.67-81, 1991.

MENDONÇA FILHO, W.F. Análise operacional de colheitadeiras florestais. Floresta e ambiente, Rio de Janeiro, UFRRJ, p.261-278. 2000.

MENEZES, J.F.; MAZIEIRO, J.V. G.; YAMASHITA, R.Y.; CORRÊA, I.M.; PASSARELA NETO, A.; FENZ, E.; MILAN, M. Avaliação de características de visibilidade apresentadas por um grupo de tratores de rodas. Campinas, SP: Instituto Agronômico de Campinas, 1985. 18p.

MINETTI, L.J.; SOUZA, A.P.; FIEDLER, N. C. Condições alimentares de operadores de motosserra de uma empresa de corte florestal no Brasil, ABERGO, 1999.

MINETTE, L.J. Análise de fatores operacionais e ergonômicos na operação de corte florestal com motosserra. 1996. 211 p. Tese (Doutorado em Ciência Florestal) - Universidade Federal de Viçosa, Viçosa, MG. 